Review Article

\section{Identifying patterns in COVID-19: Morbidity, recovery and the aftermath}

\author{
Vinod Nikhra* \\ Senior Consultant and Faculty, Department of Medicine, Hindu Rao Hospital \& NDMC Medical \\ College, New Delhi, India
}

\section{Abstract}

The infectivity and pathogenesis: SARS-CoV-2, the causative agent of Covid-19, involves Angiotensin-converting enzyme 2 (ACE2) receptors on type Il alveolar type 2 (AT2) cells in lungs. Apart from, the upper and lower respiratory tracts, the disease affects the gastrointestinal system prominently, as evidenced by the significant Gl symptoms, early in the course of the disease. In addition, the virus infects ACE2-bearing cells in other organs including the heart and blood vessels, brain, and kidneys.

Clinical features and morbidity: The clinical spectrum of COVID-19 varies from asymptomatic or pauci-symptomatic presentation to moderate to severe states characterized by respiratory failure necessitating mechanical ventilation and ICU support and those manifesting critical clinical condition with complications like sepsis, septic shock, and multiple organ dysfunction failure. The CT chest is an important tool for early identification of COVID-19 pneumonia as well as for prognostic purposes.

The recovery and residual damage: The recovery and other outcomes vary depending on age and other aspects including sex, comorbidities, and genetic factors. The outlook for older adults, who account for a disproportionate share of critical disease, is unfavorable, and most of those who survive are unlikely to return to their previous level of functioning. The disease affects their long-term health and quality of life as well as brings in propensity for truncated post-disease survival.

COVID-19 aftermath and follow up: The patients discharged from hospital following severe COVID-19, continue to suffer with lingering impact of the disease as well as that of the emergency treatments that saved their life. The post-infection reduced exercise tolerance and other subtle factors, like post viral fatigue syndrome, post-traumatic stress disorder, impaired concentration, delirium, and disturbed sleep-wake cycle often underly the functional impairment. In fact, there is need of step-down care and later a multidisciplinary support involving regular clinical assessment, respiratory review, physiotherapy, nutritional advice, and psychiatric support.

Conclusion: The life after COVID-19: After recovery from the disease, the virus SARSCoV-2, may persist for uncertain period. In addition, the chance of reinfection cannot be ruled out. The vitamin D supplementation may be helpful. In general, the quality of life (QOL) in ICU survivors improves but remains lower than general population levels, but most of the patients adapt well to their level of self-sufficiency and QOL. Also, the debility due to co-morbidities may further compromise the activity of daily living and QOL issues. The Age and severity of illness appear to be the major predictors of post-discharge physical functioning.

\section{More Information}

*Address for Correspondence: Vinod Nikhra, Consultant and Faculty, Department of Medicine Hindu Rao Hospital \& NDMC Medical College, New Delhi, India, Tel: 91-9810874937; Email: drvinodnikhra@gmail.com; drvinodnikhra@rediffmail.com

Submitted: 15 May 2020

Approved: 22 May 2020

Published: 25 May 2020

How to cite this article: Nikhra V. Identifying patterns in COVID-19: Morbidity, recovery and the aftermath. Int J Clin Virol. 2020; 4: 056-064

DOI: 10.29328/journal.ijcv.1001016

ORCiD: orcid.org/0000-0003-0859-5232

Copyright: () 2020 Nikhra V. This is an open access article distributed under the Creative Commons Attribution License, which permits unrestricted use, distribution, and reproduction in any medium, provided the original work is properly cited.

Keywords: ACE2; Acute COVID-19 cardiovascular syndrome; Acute respiratory distress syndrome; COVID-19; Cytokine storm syndrome; SARS-CoV-2

Check for updates

OPEN ACCESS

\section{The infectivity and pathogenesis}

The agent and infectivity: The causative agent of Covid-19, SARS-CoV-2 involves Angiotensin-converting enzyme 2 (ACE2) receptors on the host tissues to invade and infect. The analysis of the ACE2-RNA expression profile indicates that the ACE2-virus receptor expression is mainly concentrated in type II alveolar cells (AT2) in lungs. Further, it is more widespread in males than females, and the Asian men have a higher ACE2- expressing cell ratio than white and African American subjects [1]. In addition, the virus is able to infect ACE2-bearing cells in other organs, including the gastrointestinal tract, heart and blood vessels, and kidneys. The ACE2-expressing AT2, in addition, also express 
various other genes that positively regulate viral entry, replication and transmission. Once inside the airways, the SARS-CoV-2 through its $\mathrm{S}$ protein on viral surface, is able to recognize and stick to the ACE2 receptors, followed by the virus infecting the ACE2-bearing cells lining the upper as well as the lower respiratory tracts. With the dying cells sloughing down and filling the airways, the virus is carried deeper into the lungs as the thin layer of surfactant coating the airways becomes even thinner and the brush border less efficient to defend from the invading virus. The virus is able to transmit while still confined to the upper airways, before invading the lower respiratory tract and lungs and causing symptoms [2] The virus infects the gastrointestinal (GI) system prominently, at least in the early phase, as evidenced by the significant GI symptoms [3]. It is able to infect and multiply in the intestinal epithelial cells [4]. It appears that further intestinal invasion is effectively stalled by the fast dividing intestinal epithelial cells with help of the gut microbiota [5].

Immune Response and Pathogenesis: With the viral infection, the first response of the human body is to destroy the virus and prevent its replication. The virus invasion and intracellular replication leading to the disease manifestation, however, depends on various host factors. With the weakened immunity with aging, due to co-morbidities like cardiometabolic diseases and other factors which alter the immunity and compromise the defence system, the body is unable to stall the virus, aggravating the disease process. The infection activates the immune system leading to inflammation and pyrexia, and in severe cases, may damage the host tissues and organs. The blood vessels get inflamed and leaky, leading to pulmonary edema in lungs. In the critical disease, the SARSCoV-2 infection extensively damages the alveolar tissue and triggers an overreaction of the immune system accompanied by the excessive cytokine release, which apart from damaging the host tissues, leads to increased susceptibility to infectious bacteria, septic shock and multi-organ dysfunctions (Figure 1).

Other factors related to pathogenesis: Due to the interaction of multiple factors, which may include genderassociated and genetic linked susceptibility, the Covid-19 is a disease with variable clinical presentations, requiring a close clinical follow up, meticulous monitoring and ensuring availability of various therapeutic options including mechanical ventilatory support [6]. Such a precarious clinical scenario from virtually asymptomatic to mild to moderate, and severe to critical presentation of the disease with a high infectivity and fast changing prognosis, may make the therapeutic decisions in individual cases challenging with a high error factor on part of the care providers. The propensity to get infected themselves, adds further to the difficulties faced by the medical and paramedical workers.

\section{Clinical features and morbidity}

The clinical spectrum of COVID-19: The clinical spectrum of COVID-19 varies from asymptomatic or pauci-symptomatic presentation (Stage I) to moderate to severe states (Stage II a and IIb) characterized by respiratory failure necessitating mechanical ventilation and ICU support and those manifesting critical clinical condition (Stage III) with complications like sepsis, septic shock, and multiple organ dysfunctions (Figure 2).

The mild to moderate disease occurs in approximately in $81 \%$ of cases. The severe disease with dyspnoea and acute respiratory distress syndrome (ARDS) and lung infiltrates appearing within 48 hours occurs in $14 \%$ of cases. Whereas, the critical COVID-19 illness accompanied by respiratory failure, septic shock, and multiple organ dysfunction (MOD) or failure (MOF) has been documented in approximately $5 \%$ of cases [7].

The transition from milder symptoms to severe disease and ARDS has been related to an uncontrolled cytokine release by the hyperactive immune response. In patients, going to have the worst outcomes with COVID-19 infections, the immune system becomes overactive with excessive stimulation of $\mathrm{T}$ cells and macrophages, resulting in cytokine storm with release of a large amount of proinflammatory cytokines including interleukins (IL) - 1, 6, 12 and 18. The excessive or uncontrolled levels of cytokines released, further activate more immune cells, resulting in hyperinflammation and the cytokine release syndrome (CRS).

The clinical features of COVID-19: The most common sign is pyrexia, which is present in nearly 9 in 10 of those infected [8]. About 7 in 10 have a dry cough, of which nearly a third have cough with mucus. Tiredness and feeling of lethargy are common. Less commonly, there is sore throat, headache, muscle-aches and arthralgias. There may be nausea, vomiting or diarrhoea. Anorexia may be present, as are ageusia and anosmia. Some may complain dyspnoea, shortness of breath and a tight band wrapped around the chest. A skin rash viral exanthema, may occur especially in younger patients, as may be spots of swelling and redness. A specific finding

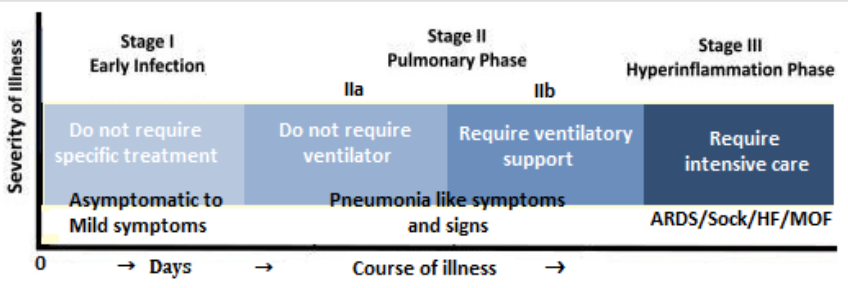

Figure 2: The spectrum and clinical stages of COVID-19. 
called Covid toes, occasionally present especially in children, is superficial small clots in blood vessels close to the skin in toes and fingers. The clinical course of the disease, in general, depends on the host's immune response. It is to be noted that the patients need a drug to boost the immune system early on in the disease, and later one to tamp it down if the disease progresses and cytokine begin to rise. The drugs that target the immune system to lower the risk of cytokine storm, may also tamp down the immune response, making it hard to clear the virus in the long run.

Lab and radiological investigations: The patients may present with lymphopenia and thrombocytopenia. There are raised CRP and elevated liver enzymes. Raised lactate dehydrogenase is a bad prognostic sign as it tends increase in cytokine storm (9). Abnormal coagulation parameters, such as elevated D-dimers and fibrinogen concentrations, and prolongation of prothrombin time (PT) and activated partial thromboplastin time (aPTT) are associated with unfavourable prognosis [10]. The radiological investigations like $\mathrm{x}$-ray and CT imaging of chest are of particular significance relating to the diagnosis, defining the extent of pulmonary involvement and disease prognosis [11].

The CT imaging in COVID-19 patients: The CT chest is an important tool for detecting pneumonia in asymptomatic or with mildly symptomatic patients with COVID-19 patients who are covert transmitters and can deteriorate clinically in a short period [12]. The typical imaging findings are ground glass opacities (GGO) with peripheral distribution, unilateral or bilateral, involving one or more lobes particularly the lower lobes, often combined with subpleural curvilinear line and fine reticulation [13]. The CT abnormalities in COVID-19 pneumonia more frequently exhibit a peripheral predominance, with less frequent pleural effusion and lymphadenopathy.

On follow up, the maximum lung involvement peaks at approximately 10 days from the onset of initial symptoms. There are 4 stages on the CT chest - Stage 1 (0-4 days): ground glass opacities; Stage-2 (5-8d days): increased crazy-paving pattern; Stage-3 (9-13days): consolidation; and Stage-4 ( $\geq 14$ days): gradual resolution of consolidation [14]. In a series of 919 patients, the CT findings in the intermediate stage of the disease were characterized by an increase in the number and size of GGOs, a progressive transformation of GGO into multifocal consolidation, with septal thickening and development of a crazy-paving pattern [15]. The presence of centrilobular nodules, mucoid impactions and unilateral segmental or lobar consolidations suggest a bacterial origin of pneumonia, or super infection.

The complications during COVID-19 illness: Often the infection caused by SARS-CoV-2 is an asymptomatic or mild disease and recover by themselves. But, approximately 1 to $5 \%$ of all COVID-19 patients are moderately severe, severe, or critically ill, and may suffer from various disease related complications (Figure 3).

\begin{tabular}{|c|c|c|c|}
\hline Complication & Inflamm. response & Structural abnormalities & Clinical outcome \\
\hline $\begin{array}{l}\text { The abnormal clotting } \\
\text { or thrombosis }\end{array}$ & $\begin{array}{l}\text { Lymphopenia } \\
\text { Thrombocytopenia } \\
\uparrow \text { IL-6, CRP } \\
\uparrow \text { D-dimer } \\
\end{array}$ & $\begin{array}{l}\text { Venous thrombosis } \\
\text { Intravascular coagulopathy } \\
\text { Myocardial injury } \\
\text { Cerebral infarction }\end{array}$ & $\begin{array}{l}\text { DVT } \\
\text { DIC } \\
\text { Myocardial infarction } \\
\text { Stroke } \\
\end{array}$ \\
\hline $\begin{array}{l}\text { Acute cardiovascular } \\
\text { syndrome (ACovCS) }\end{array}$ & $\begin{array}{l}\uparrow \text { Troponin } \\
\downarrow S T \text { or } \uparrow S T \\
\text { Arrythmias }\end{array}$ & $\begin{array}{l}\text { Wall motion abnormalities } \\
\text { De novo systolic dysfunction } \\
\text { Myocarditis or myocardiopathy }\end{array}$ & $\begin{array}{l}\text { Heart failure } \\
\text { Pericardial effusion } \\
\text { Ac coronary syndrome }\end{array}$ \\
\hline $\begin{array}{l}\text { Ac respiratory distress } \\
\text { syndrome (ARDS) }\end{array}$ & $\begin{array}{l}\text { Alveolar damage Influx } \\
\text { of inflam cells Protein } \\
\text { exudation }\end{array}$ & $\begin{array}{l}\text { Pulmonary edema } \\
\text { Pneumonia, Pleural effusion } \\
\text { Secondary bacterial infection }\end{array}$ & $\begin{array}{l}\text { Acute dyspnoea } \\
\text { Severe hypoxia } \\
\text { Respiratory failure }\end{array}$ \\
\hline $\begin{array}{l}\text { The Cytokine storm } \\
\text { syndrome (CSS) }\end{array}$ & $\begin{array}{l}\text { Macrophage activation } \\
\uparrow \uparrow \text { Cytokine release } \\
\uparrow \uparrow \text { Thrombin } \downarrow \text { Anticoag }\end{array}$ & $\begin{array}{l}\text { Activation of coagulation paths } \\
\text { Widespread Microthrombi } \\
\uparrow \text { Vascular permeability }\end{array}$ & $\begin{array}{l}\text { DIC } \\
\text { Shock } \\
\text { Multi-organ failure }\end{array}$ \\
\hline
\end{tabular}

Figure 3: Complications related to severe COVID-19 infection.

These patients need hospitalization for advanced treatment, apart from symptomatic and supportive, with antiviral drugs, antibiotics and other drugs and assisted respiratory support in a special medical facility or an intensive care unit - ICU (16). The survival odds and the potential for long-term complications, influence the decisions in ICU care, which are difficult and grey areas clinicians as well as the family members.

The abnormal clotting or thrombosis: In the severe or lethal form of COVID-19, there occur widely scattered thrombi in multiple organs early in course of the disease. The abnormal clotting plays a major role in morbidity and mortality and occurs at various sites involving small veins like in Covid toes and the large vessels in the legs leading to deep vein thrombosis (DVT), in the lungs causing to pulmonary embolism (PE) and in cerebral arteries resulting in a stroke. The etiopathology of thrombosis in COVID-19 is not fully explained, but it appears that the disease may predispose to the venous and arterial thromboembolic events due to excessive inflammation, hypoxia, immobilisation and diffuse intravascular coagulation (DIC). Added to this, is hypotension and shock, ventilator use, and multiple drug treatments themselves affecting the various organs adversely including the lungs, heart, brain, liver, and kidneys. Using medicines to prevent clotting may end up causing severe bleeding, further adding to the insult.

There has been reported a remarkably high incidence of thrombotic complications manifesting as acute PE, DVT or ischemic stroke, apart from acute coronary syndrome, myocardial infarction, and systemic arterial embolism. In a Dutch study, 31\% of patients hospitalized with COVID-19 suffered with thrombosis clots while on antiplatelets like aspirin or clopidogrel [17]. Further, in a very recent study, the US investigators led by Oxley, et al. have reported five cases of large vessel stroke over a 2-week period in COVID-19 patients under age 50 years, representing a sevenfold increase from what can be normally expected. The study links Covid-19 infection to large vessel stroke in young adults [18].

Acute COVID-19 cardiovascular syndrome (ACovCS): The ACovCS describes the impact of the disease on cardiovascular system. The ACovCS is a myocarditis-like syndrome associated with acutely reduced left ventricular 
ejection fraction in the absence of obstructive coronary artery disease. The acute myocardial injury, the fulminant myocarditis, evidenced as elevated troponin, occurs in $20 \%$ - 30\% of hospitalized Covid-19 patients [19]. The ACovCS involves demand ischemia, microvascular ischemic injury, myocarditis, acute coronary syndrome, and myocardial infarction (MI). The details of the mechanisms of ACovCS are not clear. The endomyocardial biopsy of a patient with ACovCS and cardiogenic shock showed low-grade myocardial inflammation, with localization of SARS-CoV-2 within macrophages but not cardiomyocytes. Given the exposure risks involved in these patients, the acute myocardial injury is advised to be managed conservatively, with rest and abstinence from aerobic activity for a period of 3-6 months until normalization of troponin or resolution of myocardial inflammation on magnetic resonance imaging [20].

Respiratory distress and ventilatory support: There is a high mortality rate in ventilated Covid-19 patients. In a large study, involving 3,883 COVID-19 patients, the mortality was a high as two thirds among Covidf-19 patients who required ventilation. As per the United Kingdom's Intensive Care study, $66.3 \%$ patients who required mechanical ventilation died, compared with $19.4 \%$ of the patients who required basic respiratory support. The new ICNARC (Intensive Care National Audit \& Research Centre) findings are consistent with other previous reports from smaller case series. For example, a single-center study involving 52 patients treated in Wuhan, China, showed that 37 (71\%) required mechanical ventilation and $32(61.5 \%)$ died within 28 days of ICU admission [21]. Another study from Seattle, also found that a higher proportion of COVID-19 patients, over $50 \%$, requiring mechanical ventilation died compared to about $35 \%$ patients without coronavirus who had viral pneumonia and who needed ventilation [22].

The COVID-19 pneumonia is a specific disease with dissociation between the severity of hypoxemia and the maintenance of relatively good respiratory mechanics. The lungs of COVID-19 patients tend to be more elastic and compliant than those of other ARDS patients so it is possible to allow patients to take slightly bigger breaths with a bit more tidal volume in each breath and allow them to be less sedated, more awake, and more in sync with the ventilator. They may be able to be extubated earlier. In this light, the mechanical ventilation protocols and strategies need revision and modification. It has been advocated that some COVID-19 patients might need gentler positive end-expiratory pressure because they present with an atypical form of ARDS [23]. The respiratory distress in Covid-19 is of two types, Type 1: Near normal pulmonary compliance with isolated viral pneumonia, non-ARDS patients, and Type 2: Decreased pulmonary compliance and present with ARDS.

In the Type 1, the lung's gas volume is high, the recruitability is minimal, and the hypoxemia is likely due to the loss of hypoxic pulmonary vasoconstriction and impaired regulation of pulmonary blood flow. High PEEP and prone positioning do not improve oxygenation through recruitment of collapsed areas, but redistribute pulmonary perfusion, improving the ventilation/perfusion (VA/Q) relationship. In the Type 1 patients, PEEP levels should be kept lower with higher tidal volume, the respiratory rate should not exceed 20 breaths/min, closely monitored, but too much interventions should be avoided, in general. Whereas, the Type 2 is present in $20-30 \%$ of the COVID-19 patients admitted to the ICU, manifesting severe hypoxemia is associated with compliance values $<40 \mathrm{ml} / \mathrm{cmH} 20$, indicating severe ARDS [24]. In the Type 2 patients, the standard treatment for severe ARDS should be applied with lower tidal volume, prone positioning, and relatively high PEEP.

Regarding alternate methods of advanced respiratory support, like extracorporeal membrane oxygenation (ECMO), which are special interventions for when standard therapy is not successful. The ECMO therapy is not realistic and an impractical alternative [25]. Further, it is expensive and has associated complications and a high mortality rate in patients on ECMO.

The Cytokine Storm Syndrome (CSS): The pathophysiology of CSS: As the SARS-CoV-2 virus attacks the ACE2 bearing alveolar type 2 (AT2) cells, the macrophages are recruited and increase cytokine production and attract additional immune cells to the affected area such as T-helper cells CD4 and CD8 which work to combat the infection. Pattern recognition receptors (PRRs) of the immune cells recognize the virus and signal release of the pro-inflammatory cytokines such as interferon gamma (IFN-g), tumor necrosis factors (TNFs), interleukins (ILs), and chemokines. IFN-g activates macrophages which produce IL-6, TNF- $\alpha$, and IL-10. Once the virus is cleared, the immune pathways go quiescent. In event of defective immune response to Covod-19 infection, however, this process does not shut down and leads to overproduction of proinflammatory cytokines, TNF, IL-6, and IL-1 $\beta$ causing cytokine storm. The CSS is associated with an increased vascular hyperpermeability along with activation of coagulation pathways, which predispose to the development of micro-thrombosis, disseminated intravascular coagulation (DIC), and ultimately multi-organ dysfunction [26].

Normally, thrombin promotes clot formation by activating platelets and by converting fibrinogen to fibrin. The thrombin generation is regulated by negative feedback loops and physiological anticoagulants, such as antithrombin III, tissue factor pathway inhibitor, and the protein C system. During hyper-inflammation, the feedback loops can be impaired leading to unabated production of thrombin and reduced anticoagulant concentrations due to reduced production and increased consumption. The defective procoagulantanticoagulant balance leads to the activation of coagulation pathways [27]. On the other hand, the excess thrombin further 
augments inflammation via proteinase-activated receptors (PARs), especially PAR-1. In addition, the defective immune response to COVID-19 infection predisposes to secondary bacterial infection in setting of severe COVID-19 pneumonia [28].

The patients with CSS present with, apart from a surge in IL-6, IL-1, TNF- $\alpha$ and IFN-g, elevated C-reactive protein, serum lactate dehydrogenase, D-dimer, and ferritin levels. The CSS in Covid-19 pneumonia in setting of hospitalised patients is associated with unfavourable prognosis. Various therapeutic modalities have been tried. The prophylactic use low molecular weight heparin (LMWH) may be recommended for prevention of venous thromboembolism, especially in those with significantly raised d-dimer concentrations. The LMWH has anti-inflammatory properties, which may be beneficial in COVID-19 [29]. The IL-inhibitors such as, tocilizumab (IL-1 inhibitor) may be tried, which has a large therapeutic window and a short half-life. The concentrated globulin prepared from pooled human plasma may be useful. The convalescent plasma collected from recovered COVID-19 patients is being studied as a potential treatment. The immunosuppression with corticosteroids, is controversial but may be useful in treating hyperinflammation.

\section{The recovery and residual damage}

The fatality and other severity outcomes: The CFR (case fatality rate) from Covid-19 increases with age, the overall death rate being $1.4 \%$ in those with no comorbid conditions, rises sharply to $4 \%$ for those in their 60 s, $8.6 \%$ for people in their 70 s and $13.4 \%$ for those age 80 and older. The CFR is higher among males compared to females (4.7\% vs. $2.8 \%$ ). By occupation, patients who reported being retirees had the highest CFR at $8.9 \%$. The patients with comorbid conditions had much higher rates: $13.2 \%$ for those with cardiovascular disease, $9.2 \%$ for diabetes, $8.4 \%$ for hypertension, $8.0 \%$ for chronic respiratory disease, and $7.6 \%$ for cancer. The data on the progression of disease indicate that that the time period from onset to the development of severe disease, including hypoxia, is 1 week. Among patients who have died, the time from symptom onset to outcome ranges from 2-8 weeks [30].

The recovery and other outcomes vary depending on age and other factors [31]. The outlook for older adults, who account for a disproportionate share of critical disease, is not favourable, and most of those who survive are unlikely to return to their previous level of functioning [32]. The disease affects their long-term health prospects and quality of life (QOL) as well as brings in propensity of truncated postdisease survival.

Recovery estimates for COVID-19: About 8 in 10 people who get COVID-19, are asymptomatic or suffer with mild illness. The early estimates predict that the overall COVID-19 recovery rate is between $97 \%$ and $99.75 \%$. It may take 2 weeks for to recover from illness in mild cases. For those with severe or critical cases, recovery can take up to 6 weeks. For Severe Illness need to stay in the hospital may last 2 weeks or more [33]. In general, the recovery depends on how a person's immune system responds to COVID-19. Reinfection may occur, after recovery. One early study on monkeys found that they did not get infected a second time.

According to the preliminary data from a recent UK study, involving 775 patients with COVID-19 admitted to critical care; 79 died, 86 survived and were discharged to another location, and 609 were still being treated in critical care, with uncertain prognosis [34]. Another, small study of 24 critically ill COVID-19 patients treated in Seattle hospitals, documented that over $50 \%$ died within 18 days. Of those who survived, three remained on ventilators in intensive care units, four left the ICU but stayed in the hospital, and five were discharged home. Many COVID-19 patients who need a ventilator never recover [35]. The survival rates may vary across studies and countries. A report from London's Intensive Care National Audit \& Research Centre found that $67 \%$ of reported COVID-19 patients from England, Wales, and Northern Ireland receiving advanced respiratory support died [36]. Those least likely to recover seem to be frail older patients with other pre-existing illnesses such as chronic lung disease or heart disease. The ARDS mortality is usually between $30 \%$ and $40 \%$. For older people, who tend to have more infections, mortality rates are as high as up to $60 \%$ or more.

The effect of ARDS on lungs: The COVID-19 directly impacts the lungs and damages the alveoli. There occurs damage to the wall and the lining of the alveolus and capillaries. The debris from the damage, which is plasma protein accumulates on the alveolus wall and thickens the lining and impairs the oxygen transfer to the red blood cells. As the alveoli are damaged, there occurs influx of inflamed cells and protein leak, leading to pneumonia, further impairing the oxygen intake and exchange by the lungs. The immediate assault on the body by the disease, is extensive. It targets the lungs, and through hypoxia and widespread inflammation damages the heart, kidneys, brain, and other organs. Even after coming off the ventilator, the patient often needs assistance in form of oxygen therapy, through a mask or a continuous positive airway pressure (Cpap) ventilator. The COVID-19 survivors, who have spent time on a ventilator often suffer with muscle wastage and long-term disability and may need retraining to breathe. The breathing exercises are to be advised to the recovering COVID-19 patients [37].

Although it is too early to say about lasting disabilities in COVID-19 survivors, the clues come from studies of severe pneumonia and ARDS. Though, most of the pneumonia and ARDS patients eventually recover their lung function, the conditions may lead to scarring resulting in long-term breathing problems. In addition, in the recovered patients, the prolonged inflammation seems to increase the risk of future illnesses, including coronary heart disease, stroke, and kidney 
disease. The people hospitalized for pneumonia have a risk of heart disease about four times as high as that of age-matched controls in the year after the discharge, and about 1.5 times as high in each of the next 9 years.

The disabilities and rehab measures: The older adults are at greatest risk of both severe disease and long-term impairment. The best practices for geriatric care are difficult to be followed in setting of the highly infectious disease in the hospital and ICU. During ICU care, the aim is to keep patients as lucid and mobile as possible, which can reduce the muscular weakness and allow to wean off early from the ventilator and has a positive effect on their long-term odds. The disease is highly infectious, and the rehabilitation measures can be challenging tasks.

Further, the patients who spend long time in an ICU, regardless of their illness, are prone to a set of physical, cognitive, and mental health difficulties known as postintensive care syndrome. An associated factor for hospitalized patients is risk of delirium, a state of confused thinking that can lead to long-term cognitive impairments such as memory deficits. The COVID-19, like severe acute respiratory syndrome (SARS) and Middle East respiratory syndrome (MERS), can directly damage the brain or the associated widespread inflammation can compromise the cerebral blood flow to cause neuronal deficit.

In addition, the commonly prescribe sedative drugs to suppress violent coughing and help patients tolerate the distress associated with endotracheal tube or benzodiazepines to allay anxiety can increase the risk and intensity of delirium. To overcome this and improve patient care and outcomes, many ICUs, now adopt a frequent interruption of narcotics and sedatives plus a decrease of ventilator pressure to test whether patients can wake up, breathe, and tolerate the ventilator without drugs. This practice, of course, requires close monitoring and risk of exposure to the Covid-19 infection. Early reports from the ICUs care, suggested that the Covid-19 patients should be put on ventilators early in the course of the disease. But there are the downsides of early ventilation [38]. It may be possible to differentiate the subtypes and stage of respiratory failure in COVID-19 patients, who are more likely to need to ventilatory support.

Post-recovery complications: There are immediate as well as the delayed post-recovery complications. Those patients who have suffered with high fevers or severe diarrheal illness can remain mildly dehydrated and suffer with electrolytes disturbance, and various fallouts. They may not tolerate nutrition as well and get suboptimal nutrition and suffer with weight loss and emaciation. Frailty is common, associated with ongoing fatigue and lethargy. The long-term complications include myocarditis and cardiomyopathy, which can exacerbate their propensity to coronary artery disease. There occurs an increased risk for future illnesses, including
MI, stroke, and kidney disease. With other pre-existing ilnesses like diabetes, there is more risk for complications.

In addition, the underlying conditions like chronic obstructive pulmonary disease (COPD) and other chronic lung diseases are exacerbated. Some of these patients may have significant scarring of the lungs and suffer with reduced lung function. Those who remain for a long period on a ventilator are prone to respiratory and extra-respiratory muscle atrophy and weakness. Their immune system is less robust, and they are prone to infections. In addition, many of them may suffer with post-traumatic stress disorder (PTSD), impaired concentration, delirium and disturbed sleep-wake cycle afterwards. A study of people hospitalized for SARS found that more than one-third had moderate to severe symptoms of depression and anxiety one year later [39].

\section{COVID-19 aftermath and follow up}

The post-discharge period: The patients hospitalized with severe COVID-19, after discharge suffer with lingering impact of the disease as well as that of the emergency treatments that allowed them to survive it. The post-infection reduced exercise tolerance suggests that there may be more subtle factors, like post viral fatigue, underlying the functional impairment. In fact, there is need of step-down care while in the hospital before discharge to home or the community care with primary care support [40]. Later, the additional inputs are likely to be multidisciplinary for regular clinical assessment, recomposing respiratory review, physiotherapy, nutritional advice, and psychiatric support as the discharged patients regain their health.

The post-discharge follow up: About $50 \%$ of patients admitted to hospital will require no further input on discharge. $45 \%$ will need some form of low level medical or social input for recovery, and a predicted $5 \%$ of patients will require more focused, ongoing intense rehabilitation (Figure 4).

In general, a combination of physical interventions such physiotherapy and graded exercise programmes, good nutrition and clinically validated mental health support interventions such as cognitive-behavioral therapy (CBT) focusing on changing the automatic negative thoughts that can contribute to and worsen emotional difficulties, depression, and anxiety, and possibly antidepressants may provide

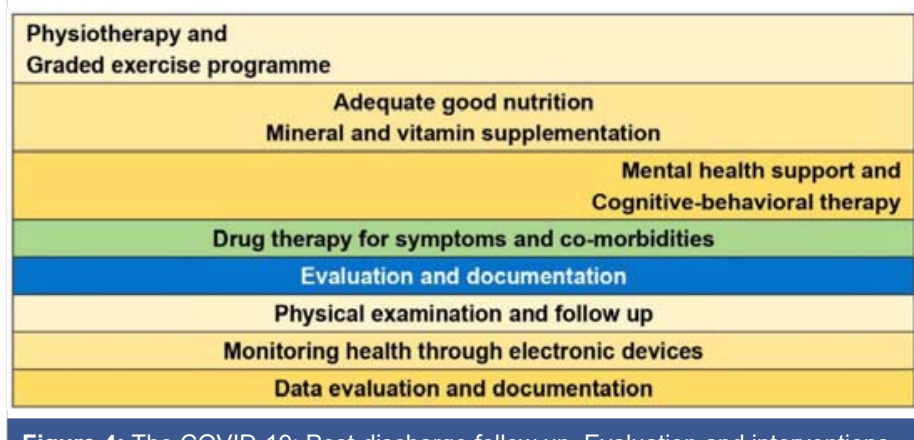

Figure 4: The COVID-19: Post-discharge follow up, Evaluation and interventions. 
benefits. A designed care approach for severe COVID-19 discharged patients may include monitoring them using computers and smartphones and treating them remotely in hopes of preventing readmission to the hospital, and planning visits at home on case to case basis.

A two year follow up study involving 106 patients aged 80 years or over, has documented that $40(37.7 \%)$ died in the ICU and $66(62.2 \%)$ patients were discharged alive from the ICU. Out of this, 25 patients died before the one-year evaluation. Of the 33 survivors at one year, seven refused the evaluation. of the 26 remaining patients, three had dementia and the self-sufficiency could be assessed by the relatives. QOL was assessed in the rest, who were found to have significantly higher scores for psychological health, social relationships, environment, fear of death and dying, expectations about past, present, and future activities, and intimacy (friendship and love). Of the 23 patients, 18 agreed for another ICU admission should the need arise [41].

\section{Conclusion}

\section{The life after COVID-19}

The Recovery and SARS-CoV-2 virus: During the recovery period, the viral particles can be found not only in the nasal passages, throat, and respiratory tract, but also in tears, stool, the kidneys, liver, pancreas, and heart. They have been found in the cerebrospinal fluid (CSF) in a patient with meningitis. The virus could persist in the body for up to two weeks or more after symptoms had vanished. The virus has been shown to survive in a Chinese patient's respiratory tract for 37 days, well above the average of 24 days for those with critical disease status [42]. There are further reports from China that $14 \%$ of recovering patients were retested positive [43]. It is possible that the virus may persist as a latent infection, like chickenpox, lying dormant in the body, re-emerging periodically as shingles, or become a chronic infection, like hepatitis B, living within the body for a sustained period of time, causing long-term damage.

Recovery from COVID-19 and vitamin D: Vitamin D levels are often low in the aging population, which is also the most vulnerable group of population for COVID-19. Vitamin $\mathrm{D}$ has been shown to protect against acute respiratory infections [44]. A team of researchers have found the link between low levels of vitamin D and COVID-19 mortality rates across Europe. They found that levels of vitamin D among citizens of 20 countries in Europe was strongly associated with the mortality caused by Covid-19 and advised vitamin D supplementation to protect against COVID-19 infection [45].

Post-discharge oeriod: Things that matter: The human lifespan has increased across the globe as a result of economic progress, technological advances, and improved healthcare. Simultaneously, there has been a steady increase in the proportion of elderly individuals in different societies. As the natural fallout of a growing number of elderly and very elderly patients are being admitted to the ICU. Compared with the general population, ICU survivors report lower QOL prior to ICU admission. After hospital discharge, QOL in ICU survivors improves but remains lower than general population levels. Age and severity of illness are major predictors of physical functioning [46].

The critical care seeks to ensure survival as well as restore to the pre-admission level of function and QOL. Few data are available on QOL in elderly and very elderly ICU survivors compared to the general population. The study by Tabah, et al. inferred that in the highly selective cohort of the elderly patients one year after ICU discharge, the patients were satisfied with their level of self-sufficiency and QOL [41]. Further, the QOL, physical health, sensory abilities, selfsufficiency, and social participation had slightly lower ratings than other domains like social relationships, environment, and death related issues.

\section{References}

1. Zhao $Y$, Zhao Z, Wang $Y$. Single-cell RNA expression profiling of ACE2, the receptor of SARS-CoV-2. 2020.

2. Guo YR, Cao QD, Hong ZS, Tan YY, Chen SD, et al. The origin, transmission and clinical therapies on coronavirus disease 2019 (COVID-19) outbreak - an update on the status. Mil Med Res. 2020; 7: 11. PubMed: https://www.ncbi.nlm.nih.gov/pubmed/32169119

3. Tian Y, Rong L, Nian W, He Y. Review article: gastrointestinal features in COVID-19 and the possibility of faecal transmission. Alimentary Pharm \& Thera. 2020; 51: 843-851.

https://onlinelibrary.wiley.com/doi/abs/10.1111/apt.15731?af=R

4. Lamers MM, Beumer J, van der Vaart J, et al. SARS-CoV-2 productively infects human gut enterocytes. Science. 2020.

PubMed: https://www.ncbi.nlm.nih.gov/pubmed/32358202

5. Nikhra V. The Trans-zoonotic Virome interface: Measures to balance, control and treat epidemics. Ann Biomed Sci Eng. 2020; 4: 020-027.

6. Guan WJ, Ni ZY, Hu Y, et al. For the China Medical Treatment Expert Group for Covid-19. Clinical Characteristics of Coronavirus Disease 2019 in China. N Engl J Med. 2020; 382: 1708-1720.

7. McIntosh K, Hirsch MS, Bloom A. Coronavirus disease 2019 (COVID-19): Epidemiology, virology, clinical features, diagnosis, and prevention. Literature review current through: Mar 2020, last updated: 2020. https://www.uptodate.com/contents/coronavirus-disease-2019covid-19-epidemiology-virology-clinical-features-diagnosis-andprevention

8. Fu L, Wang B, Yuan T, et al. Clinical characteristics of coronavirus disease 2019 (COVID-19) in China: A systematic review and metaanalysis. J Infect. 2020,

PubMed: https://www.ncbi.nlm.nih.gov/pmc/articles/PMC7151416/

9. Petrilli CM, Jones SA, Yang $\mathrm{J}$, et al. Factors associated with hospitalization and critical illness among 4,103 patients with COVID-19 disease in New York City. Intensive Care and Critical Care Medicine. 2020.

10. Tang N, Li D, Wang X, Sun Z. Abnormal coagulation parameters are associated with poor prognosis in patients with novel coronavirus pneumonia. J Thromb Haemost. 2020; 18: 844-847. PubMed: https://www.ncbi.nlm.nih.gov/pubmed/32073213 
11. Rodriguez-Morales AJ, Cardona-Ospina JA, Gutiérrez-Ocampo E, et al. Clinical, laboratory and imaging features of COVID-19: A systematic review and meta-analysis. Travel Med Infect Dis. 2020; 101623. PubMed: https://www.ncbi.nlm.nih.gov/pubmed/32179124

12. Meng $\mathrm{H}$, Xiong $\mathrm{R}$, He R, Lin $\mathrm{W}$, Hao $\mathrm{B}$, et al. CT imaging and clinical course of asymptomatic cases with COVID-19 pneumonia at admission in Wuhan, China. J Infect. 2020.

PubMed: https://www.ncbi.nlm.nih.gov/pubmed/32294504

13. Hani C, Trieu NH, Saab I, S. Dangeard,a S. Bennani, et al. Thoracic imaging COVID-19 pneumonia: A review of typical CT findings and differential diagnosis. Diagnostic and Interventional Imaging. 2020; 101: 263-268.

PubMed: https://www.ncbi.nlm.nih.gov/pmc/articles/PMC7129663/

14. Pan F, Ye T, Sun P, Gui S, Liang B. et al. Time Course of Lung Changes on Chest CT During Recovery From 2019 Novel Coronavirus (COVID-19). Radiology. 2020; 295: 715-721.

PubMed: https://www.ncbi.nlm.nih.gov/pubmed/32053470

15. Salehi S, Abedi A, Balakrishnan S, Gholamrezanezhad A. Coronavirus disease 2019 (COVID-19): a systematic review of imaging findings in 919 Patients. AJR Am J Roentgenol. 2020; 1-7.

PubMed: https://www.ncbi.nlm.nih.gov/pubmed/32174129

16. Murthy S, Gomersall CD, Fowler RA. Care for Critically III Patients with COVID-19. JAMA. 2020; 323: 1499-1500.

PubMed: https://www.ncbi.nlm.nih.gov/pubmed/32159735

17. Klok FA, Kruip MJHA, van der Meer NJM, Arbous MS, Gommers DAMPJ, et al. Incidence of thrombotic complications in critically ill ICU patients with COVID-19. Thromb Res. 2020;

PubMed: https://www.ncbi.nlm.nih.gov/pubmed/32291094

18. Oxley TJ, Mocco J, Majidi S, Kellner CP, Shoirah H, et al. Large-Vessel Stroke as a Presenting Feature of Covid-19 in the Young. NEJM Corres. 2020.

PubMed: https://www.ncbi.nlm.nih.gov/pubmed/32343504

19. Chen C, Zhou Y, Wang DW. SARS-CoV-2: a potential novel etiology of fulminant myocarditis. Herz. 2020; 45: 230-232.

PubMed: https://pubmed.ncbi.nlm.nih.gov/32140732/

20. Hendren NS, Drazner MH, Bozkurt B, Cooper LT, Jr. Description and Proposed Management of the Acute COVID-19 Cardiovascular Syndrome. Circulation. 2020.

21. Yu Y, Shu H, Xia J, Shu H, Xia J, et al. Clinical course and outcomes of critically ill patients with SARS-CoV-2 pneumonia in Wuhan, China: a single-centered, retrospective, observational study. Lancet Resp Med. 2020; 8: P475-481.

PubMed: https://pubmed.ncbi.nlm.nih.gov/32105632/

22. Bhatraju PK, Ghassemieh BJ, Nichols M, Kim R, Jerome KR, et al Covid-19 in Critically III Patients in the Seattle Region - Case Series. NEJM. 2020.

PubMed: https://www.ncbi.nlm.nih.gov/pubmed/32227758

23. Gattinoni L, Chiumello D, Rossi S. COVID-19 pneumonia: ARDS or not?. Crit Care. 24; 154. 2020.

PubMed: https://www.ncbi.nlm.nih.gov/pubmed/32299472

24. Maiolo G, Collino F, Vasques F, Rapetti F, Tonetti T, et al. Reclassifying acute respiratory distress syndrome. Am J Respir Crit Care Med. 2018; 197: 1586-1595.

PubMed: https://www.ncbi.nlm.nih.gov/pubmed/29345967

25. Henrya BM, Lippib G. Poor survival with extracorporeal membrane oxygenation in acute respiratory distress syndrome (ARDS) due to coronavirus disease 2019 (COVID-19): Pooled analysis of early reports. J Crit Care. 2020.

PubMed: https://www.ncbi.nlm.nih.gov/pmc/articles/PMC7118619/
26. Porter DL, Maloney DG. Cytokine release syndrome (CRS). UpToDate. https://www.uptodate.com/contents/cytokine-release-syndrome-crs?

27. Jose RJ, Manuel A. COVID-19 cytokine storm: the interplay between inflammation and coagulation. Lancet Resp Med Corres. 2020, PubMed: https://www.ncbi.nlm.nih.gov/pubmed/32353251

28. Tang N, Li D, Wang X, Sun Z. Abnormal coagulation parameters are associated with poor prognosis in patients with novel coronavirus pneumonia. J Thromb Haemost. 2020; 18: 844-847. PubMed: https://www.ncbi.nlm.nih.gov/pubmed/32073213

29. Tang N, Huan B, Xing C, Gong J, Li D, et al. Anticoagulant treatment is associated with decreased mortality in severe coronavirus disease 2019 patients with coagulopathy. J Thromb Haemost. 2020; 18: 1094 1099.

PubMed: https://www.ncbi.nlm.nih.gov/pubmed/32220112

30. Report of the WHO-China Joint Mission on Coronavirus Disease 2019 (COVID-19). 2020. https://www.who.int/publications-detail/report-ofthe-who-china-joint-mission-on-coronavirus-disease-2019

31. Porcheddu R, Serra C, Kelvin D, Kelvin N, Rubino S. Similarity in Case Fatality Rates (CFR) of COVID19/SARS-COV-2 in Italy and China. J Infect Dev Ctries. 2020; 14: 125-128.

PubMed: https://www.ncbi.nlm.nih.gov/pubmed/32146445

32. Richardson S, Hirsch JS, Narasimhan M, Crawford JM, McGinn T, et al. Presenting Characteristics, Comorbidities, and Outcomes Among 5700 Patients Hospitalized With COVID-19 in the New York City Area. JAMA. 2020.

PubMed: https://www.ncbi.nlm.nih.gov/pubmed/32320003

33. Zhou F, Yu T, Du R, Fan G, Liu Y, et al. Clinical course and risk factors for mortality of adult inpatients with COVID19 in Wuhan, China: a retrospective cohort study. The Lancet. 2020; 395: 1054-1062. PubMed: https://www.ncbi.nlm.nih.gov/pubmed/32171076

34. Perez-Guzman PNP, Daunt A, Mukherjee S. Report 17: Clinical characteristics and predictors of outcomes of hospitalised patients with COVID-19 in a London NHS Trust: a retrospective cohort study. Imperial College COVID-19 response team. 2020.

35. Vincent JL, Taccone FS. Understanding pathways to death in patients with COVID-19. Lancet Respir Med. 2020; 8: 430-432.

PubMed: https://www.ncbi.nlm.nih.gov/pubmed/32272081

36. Harrison DA, Parry GJ, Carpenter JR, Short A, Rowan K. A new risk prediction model for critical care: The Intensive Care National Audit \& Research Centre (ICNARC) model. Crit Care Med. 2007; 35: 1091 1098.

PubMed: https://www.ncbi.nlm.nih.gov/pubmed/17334248

37. Which breathing techniques help with COVID-19? Official $Q$ \& A. Medically reviewed by Drugs.com https://www.drugs.com/medicalanswers/breathing-techniques-covid-19-3536315/

38. Harman EM. Ed. Pinsky MR. What are the disadvantages to mechanical ventilation in the treatment of acute respiratory distress syndrome (ARDS)? Drugs \& Diseases > Critical Care > Acute Respiratory Distress Syndrome (ARDS) Q\&A. 2020. https://www.medscape.com/ answers/165139-43338/what-are-the-disadvantages-to-mechanicalventilation-in-the-treatment-of-acute-respiratory-distress-syndromeards.

39. Mak IWC, Chu CM, Pan PC, Yiu MGC, Chan VL. Long-term psychiatric morbidities among SARS survivors. Gen Hosp Psychiatry. 2009; 31: 318-326.

PubMed: https://www.ncbi.nlm.nih.gov/pubmed/19555791

40. Guidance for stepdown of infection control precautions within hospitals and discharging COVID-19 patients from hospital to home settings. 2020. https://icmanaesthesiacovid-19.org/news/stepdown-of-infectioncontrol-precautions-and-discharging-covid-19-patients-to-homesettings. 
41. Tabah A, Philippart F, Timsit JF, Willems V, Français A, et al. Quality of life in patients aged 80 or over after ICU discharge. Crit Care. 2010; 14: R2. PubMed: https://www.ncbi.nlm.nih.gov/pubmed/20064197

42. Zhou F, Yu T, Du R, Fan G, Liu Y, et al. Clinical course and risk factors for mortality of adult inpatients with COVID-19 in Wuhan, China: a retrospective cohort study. Lancet. 2020; 395: 1054-1062.

PubMed: https://www.ncbi.nlm.nih.gov/pubmed/32171076

43. Chen Y, Bai W, Liu B, et al. Re-evaluation of Nucleic Acid Retested Positive Cases in the Recovered COVID-19 Patients: Report from a Designated Transfer Hospital in Chongqing, China. 2020.

44. Martineau AR, Jolliffe DA, Greenberg L, Greenberg L, Aloia JF, et al.
Vitamin D supplementation to prevent acute respiratory infections: individual participant data meta-analysis. Health Technol Assess. 2019; 356: i6583.

PubMed: https://pubmed.ncbi.nlm.nih.gov/28202713/

45. Ilie PC, Stefanescu S, Smith L. The role of Vitamin D in the prevention of Coronavirus Disease 2019 infection and mortality. Research Squqre. 2020.

46. Dowdy DW, Eid MP, Sedrakyan A, Mendez-Tellez PA, Pronovost PJ, et al. Quality of life in adult survivors of critical illness: a systematic review of the literature. Intensive Care Med. 2005' 31: 611-620. PubMed: https://www.ncbi.nlm.nih.gov/pubmed/15803303nces 